Mathematical Modelling AND ANALysis

Volume 18 Number 4, September 2013, 505-528

http://dx.doi.org/10.3846/13926292.2013.840866

(c) Vilnius Gediminas Technical University, 2013
Publisher: Taylor\&Francis and VGTU

http://www.tandfonline.com/TMMA

Print ISSN: 1392-6292

Online ISSN: 1648-3510

\title{
A Stage-Structured Predator-Prey SI Model with Disease in the Prey and Impulsive Effects
}

\author{
Tongqian Zhang ${ }^{a}$, Xinzhu Meng ${ }^{a}$, Yi Song ${ }^{a}$, \\ Tonghua Zhang ${ }^{b}$ \\ ${ }^{a}$ Shandong University of Science and Technology \\ 266590 Qingdao, China \\ ${ }^{b}$ Swinburne University of Technology \\ PO Box 218, Hawthorn, 3122 VIC, Australia \\ E-mail: zhangtongqian@sdust.edu.cn \\ E-mail(corresp.): mxz721106@sdust.edu.cn \\ E-mail: songyi012@yahoo.cn; tonghuazhang@swin.edu.au
}

Received November 11, 2011; revised July 22, 2013; published online September 1, 2013

\begin{abstract}
This paper aims to develop a high-dimensional SI model with stage structure for both the prey (pest) and the predator, and then to investigate the dynamics of it. The model can be used for the study of Integrated Pest Management (IPM) which is a combination of constant pulse releasing of animal enemies and diseased pests at two different fixed moments. Firstly, we use analytical techniques for impulsive delay differential equations to obtain the conditions for global attractivity of the 'pest-free' periodic solution and permanence of the population model. It shows that the conditions strongly depend on time delay, impulsive release of animal enemies and infective pests. Secondly, we present a pest management strategy in which the pest population is kept under the economic threshold level (ETL) when the pest population is permanent. Finally, numerical analysis is presented to illustrate our main conclusion.
\end{abstract}

Keywords: age-structured population, delay, impulsive differential equation, global asymptotic stability, predator-prey model.

AMS Subject Classification: 34B10.

\section{Introduction}

Large-scale pest outbreak may bring serious ecological and economic problems to our society. For example, cotton bollworm outbreaks have caused severe losses of cotton in Xinjiang, Henan, Shandong, Hebei provinces of China in the past two decades; outbreaks of large-scale locusts, which feed on leaves, stems of crops, can cause serious ecological disaster. Organic chemicals (chemical insecticides) have been used to control bands and swarms for more than half a century. It turns out to be useful since they can quickly kill a significant portion 
of a pest population. Extensive use of chemical pesticides greatly enhances the human ability to control insects, however, it also brought well-known issues of environmental pollution, ecological balance, food safety, and so on. Pesticide caused environmental pollution is believed to be the number one killer to human health and other creatures. Therefore, how effectively and without compromise of environment to control insects has become an increasingly complex issue over the past two decades.

A pest control problem is a problem of population dynamics. It is proved to be more effective to control the pest by biological technologies, which include using predatory natural enemies, microbial, parasitic enemies, etc. It can be cultivated in biological laboratory or natural environment. This method is mostly welcomed by the people because it is harmless not only to environment and human health, but also the development of population. Nowadays, this method has been widely applied in the pest control of vegetables, fruits, some trees in agriculture and planting. In recent years, impulsive systems are found in many research areas of applied sciences $[7,10,15,19,21]$. Impulsive delay differential equations play a significant role in various branches of applied sciences including biology and population dynamics. Generally, the theory of impulsive delay differential equations is relatively mature $[9,13]$ and the introduction of time delay and impulse to predator-prey models with stage-structure enriches the biological background greatly. But the consequence is obvious because the system becomes nonautonomous and quite complicated. Recently, twodimensional delayed stage-structured models with impulsive effect at one fixed impulsive moment have been investigated in $[5,16]$. The predator-prey models with stage structure for the predator have been extensively investigated, $[4,20]$ to name but a few. In the real world, as immature prey takes $\tau$ units of time to mature, it is necessary to consider the death toll during the juvenile period. Hence time delay is endowed with more vital significance in stage-structured models, for example $[1,6,17]$.

In this paper, we aim to propose a pest control SI model which is stagestructured for both the pest and the predator by introducing a constant periodic releasing animal enemies and infective pests at two different fixed moments. As the diseased and juvenile individuals of pests cause damage to crops very little, we assume in our model natural enemies prey on mature pests, but the diseased and juvenile pests, and we only need to control the adult pest (prey). Then we propose a high-dimensional delayed predator-prey model with two stage structures and two different fixed moment impulse effects. New technique is also developed to investigate the dynamics of the model.

\subsection{Model formulation}

The basic model considered is based on the following SI epidemic model

$$
\left\{\begin{array}{l}
\dot{S}(t)=b S(t)-\beta S(t) I(t)-d S(t), \\
\dot{I}(t)=\beta S(t) I(t)-d I(t)-r I(t),
\end{array}\right.
$$

where $S(t)$ and $I(t)$ denote the members of the population susceptible to the disease, and the infective members, respectively. Aiello and Freedman intro- 
duced the following famous model [1]:

$$
\left\{\begin{array}{l}
\dot{x}_{j}(t)=\alpha x(t)-\gamma x_{j}(t)-\alpha e^{-\gamma \tau} x(t-\tau), \\
\dot{x}(t)=\alpha e^{-\gamma \tau} x(t-\tau)-e x^{2}(t),
\end{array}\right.
$$

where they assumed for the mature population that the death rate is of a logistic nature, that is, proportional to the square of the population with proportionality constant $e$. Meng and Chen introduced a stage-structured SI ecoepidemiological model with time delay and impulsive controlling [14]. Cooke [3] formulated an SIR model with time delay effect by assuming that the force of infection at time $t$ is given by $\beta S(t) I(t-\tau)$, where $\beta$ is the average number of contacts per infective per day and $\tau>0$ is a fixed time during which the infectious agents develop in the vector and it is only after that time that the infected vector can infect a susceptible human. Levin et al. adopted a incidence form like $\beta S^{p}(t) I^{q}(t), \beta S^{p}(t) I^{q}(t) / N, p, q \in R_{+}$which depends on different infective diseases and environments [12]. We shall consider the case of $p=1, q=2$, $N=1$, i.e. $\beta S(t) I^{2}(t)$. Motivated by recent work, we formulate the following delayed pest management SI model with stage structure for both the pests and the predators, and the effects of constant natural enemies and diseased pests impulsive input periodically at two different fixed moments:

$$
\left\{\begin{aligned}
\frac{d S_{j}(t)}{d t}= & r S(t)-d_{1} S_{j}(t)-r e^{-d_{1} \tau} S(t-\tau), \quad t \neq(n+l-1) T, t \neq n T, \\
\frac{d S(t)}{d t}= & r e^{-d_{1} \tau} S(t-\tau)-d_{2} S^{2}(t) \\
& -\beta S(t) I^{2}(t)-\frac{\alpha S(t) y(t)}{1+\omega y(t)}, \quad t \neq(n+l-1) T, t \neq n T, \\
\frac{d I(t)}{d t}= & \beta S(t) I^{2}(t)-d_{3} I(t), \quad t \neq(n+l-1) T, t \neq n T, \\
\frac{d y_{j}(t)}{d t}= & \frac{\lambda \alpha S(t) y(t)}{1+\omega y(t)}-\left(m+d_{4}\right) y_{j}(t), \quad t \neq(n+l-1) T, t \neq n T, \\
\frac{d y(t)}{d t}= & m y_{j}(t)-d_{5} y(t), \quad t \neq(n+l-1) T, t \neq n T, \\
\Delta S_{j}(t)= & 0, \quad \Delta S(t)=0, \quad \Delta I(t)=0, \quad \Delta y_{j}(t)=q, \quad \Delta y(t)=0, \\
t=(n+l-1) T, &
\end{aligned}\right.
$$

where $S_{j}(t)$ and $S(t)$ represent the density of the immature and mature pest, $y_{j}(t)$ and $y(t)$, the density of the immature and mature natural enemy, respectively; $I(t)$ represents the density of the diseased pest at time $t, r$ is the growth rate of the mature pest in the absence of the predator, $d_{1}, \mu, d_{3}, d_{4}$ and $d_{5}$ are the death rates of the immature pest, mature pest infective pest, immature natural enemy and mature natural enemy. $d_{2}$ is the mature pest death and overcrowding rate. $\beta$ is the infection rate of infective pest, $\alpha$ is the predation rate of the predator, $\lambda$ represents the conversion rate at which ingested prey in 
excess of what is needed for maintenance is translated into predator population increase, $\omega$ measures the psychological or inhibitory effect respectively; $\tau$ is the mean length of the juvenile period; $T$ is the period of the impulsive effect; $q$ is the released amount of natural enemies at every impulsive period $(n+l-1) T$; $p$ represents the released amount of infective pests at every impulsive period $n T$. From the point of biology, we only consider system (1.1) in the following region: $D=\left\{\left(S_{j}, S, I, y_{j}, y \mid S_{j}, S, I, y_{j}, y \geq 0\right\}\right.$. Let

$C^{+}=\left\{\phi=\left(\phi_{1}(s), \phi_{2}(s), \phi_{3}(s), \phi_{4}(s), \phi_{5}(s)\right) \in C: \phi_{i}(0)>0(i=1,2,3,4,5)\right\}$,

where $\phi_{i}(s)$ is non-negative, bounded and continuous function for $s \in[-\tau, 0]$. $C$ denotes the Banach space $C\left([-\tau, 0], R^{5}\right)$ of continuous functions mapping the interval $[-\tau, 0]$ into $R^{5}$. Motivated by the application of systems (1.1) to population dynamics, we assume that solutions of systems (1.1) satisfy the initial conditions

$$
\phi \in C^{+} .
$$

For continuity of initial conditions, we require

$$
\phi_{1}(0)=\int_{-\tau}^{0} r e^{d_{1} \sigma} S(\sigma) d \sigma .
$$

Since the variables $S_{j}(t)$ only appear in the first equation of system (1.1), we only need to consider the subsystem of system (1.1) as follows:

$$
\left\{\begin{aligned}
& \frac{d S(t)}{d t}= r e^{-d_{1} \tau} S(t-\tau)-d_{2} S^{2}(t) \\
&-\beta S(t) I^{2}(t)-\frac{\alpha S(t) y(t)}{1+\omega y(t)}, \quad t \neq(n+l-1) T, \quad t \neq n T, \\
& \frac{d I(t)}{d t}= \beta S(t) I^{2}(t)-d_{3} I(t), \quad t \neq(n+l-1) T, \quad t \neq n T, \\
& \frac{d y_{j}(t)}{d t}= \frac{\lambda \alpha S(t) y(t)}{1+\omega y(t)}-\left(m+d_{4}\right) y_{j}(t), \quad t \neq(n+l-1) T, t \neq n T, \\
& \frac{d y(t)}{d t}= m y_{j}(t)-d_{5} y(t), \quad t \neq(n+l-1) T, t \neq n T, \\
& \Delta S(t)= 0, \quad \Delta I(t)=0, \quad \Delta y_{j}(t)=q, \quad \Delta y(t)=0, \quad t=(n+l-1) T, \\
& \Delta S(t)=0, \quad \Delta I(t)=p, \quad \Delta y_{j}(t)=0, \quad \Delta y(t)=0, \quad t=n T .
\end{aligned}\right.
$$

\subsection{Boundedness}

First we should point out the solution of (1.1), $x(t)=\left(S_{j}(t), S(t), I(t), y_{j}(t)\right.$, $y(t))$ is a piecewise continuous function on $(n \tau,(n+1) \tau]$, and $x\left(n \tau^{+}\right)=$ $\lim _{t \rightarrow n \tau^{+}} x(t)$. For system (1.1) to be biologically meaningful, it is important to prove that all its state variables are non-negative for all time. In other word, solutions of system (1.1) with positive initial value remain positive for all time $t>0$. 
Let $S_{j}(0)>0, S(0)>0, I(0)>0, y_{j}(0)>0$ and $y(0)>0$. Then, one can easily prove that the solutions $\left(S_{j}, S, I, y_{j}, y\right)$ of system (1.1) are positive for all $t>0$. First, we show that $S(t)>0$ for all $t>0$. Notice $S(0)>0$, hence if there exists $t_{0}$ such that $S\left(t_{0}\right)=0$, then $t_{0}>0$. Assume that $t_{0}$ is the first such time that $S(t)=0$, that is $t_{0}=\inf \{t>0 \mid S(t)=0\}$; then from the second equation of (1.1) we get

$$
S^{\prime}\left(t_{0}\right)=r e^{-d_{1} \tau} S(t-\tau)>0 .
$$

Hence for sufficiently small $\varepsilon>0, S^{\prime}\left(t_{0}-\varepsilon\right)>0$. But by the definition of $t_{0}$, $S^{\prime}\left(t_{0}-\varepsilon\right) \leq 0$. This contradiction shows that $S(t)>0$ for all $t>0$.

From the third equation of (1.1) we get for $t \neq n T$, that

$$
I(t)=I(0) e^{\int_{0}^{t}\left(\beta S(t) I(t)-d_{3}\right) d t} .
$$

For $t=n T$, we have $I\left(n T^{+}\right)=I(n T)+p, p \geq 0$, it is easy to see that $I(t)>0$ for all $t>0$. From the fourth equation of (1.1) for $t \neq(n+l-1) T$, we have that

$$
y_{j}(t)=y_{j}(0) e^{\int_{0}^{t}\left(\frac{\lambda \alpha y(t)}{y_{j}(t)}-m-d_{4}\right) d t}
$$

and for $t=(n+l-1) T$, we have $y_{j}\left(n T^{+}\right)=y_{j}(n T)+q, q \geq 0$, thus we have $y_{j}(t)>0$ for all $t>0$. Similarly from the fifth equation of (1.1), we get for $t>0$ that

$$
y(t)=y(0) e^{\int_{0}^{t}\left(\frac{m y_{j}(t)}{y(t)}-d_{5}\right) d t} .
$$

Obviously we have $y_{j}(t)>0$ for all $t>0$.

Finally we consider the following equation:

$$
u^{\prime}(t)=-r e^{-d_{1} \tau} S(t-\tau)-d_{1} u(t)
$$

and comparing with (1.1), we note that if $u(t)$ is the solution of (1.4) and if $S_{j}(t)>0$ can solve (1.1), then $S_{j}(t)>u(t)$ for $0 \leq t \leq \tau$. Solving (1.4) gives

$$
u(t)=e^{-d_{1} t}\left(S_{j}(0)-\int_{0}^{t} r e^{d_{1}(\sigma-\tau)} S(\sigma-\tau) d \sigma\right) .
$$

From $\phi_{1}(0)=\int_{-\tau}^{0} r e^{d_{1} \sigma} S(\sigma) d \sigma$, one can obtain

$$
u(\tau)=e^{-d_{1} \tau}\left(\int_{-\tau}^{0} r e^{d_{1} \sigma} S(\sigma) d \sigma-\int_{0}^{\tau} r e^{d_{1}(\sigma-\tau)} S(\sigma-\tau) d \sigma\right) .
$$

Notice that $\int_{-\tau}^{0} r e^{d_{1} \sigma} S(\sigma) d \sigma$ is equivalent to $\int_{0}^{\tau} r e^{d_{1}(\sigma-\tau)} S(\sigma-\tau) d \sigma$. And we obtain $u(\tau)=0$. Hence $S_{j}(\tau)>0$. Since $u(t)$ is strictly decreasing, $S_{j}(t)>$ $u(t)>0$ for $0 \leq t \leq \tau$. So $S_{j}(t)>0$ for $0 \leq t \leq \tau$.

We now show by induction that $S_{j}(t)$ is positive on $n \tau<t<(n+l) \tau$, $n=0,1, \ldots$. We have just shown that this is valid for $n=0$. Assume it is valid for $n=0,1, \ldots, k-1$. Then $S_{j}(k \tau)>0$. Consider the equation given by

$$
u^{\prime}(t)=-r e^{-d_{1} \tau} S(t-\tau)-d_{1} u(t), \quad u(k \tau)=y_{j}(k \tau) .
$$


From (1.1), $S_{j}(t)>u(t)$ for $t>k \tau$. Now (1.5) is equivalent to the integral equation

$$
u(t)=e^{-d_{1}(t-k \tau)}\left(S_{j}(k \tau)-\int_{k \tau}^{t} r e^{d_{1}(\sigma-k \tau-\tau)} S(\sigma-\tau) d \sigma\right) .
$$

Hence

$$
u((k+1) \tau)=e^{-d_{1} \tau}\left(S_{j}(k \tau)-\int_{k \tau}^{(k+1) \tau} r e^{d_{1}(\sigma-(k+1) \tau)} S(\sigma-\tau) d \sigma\right) .
$$

From $\phi_{1}(0)=\int_{-\tau}^{0} r e^{d_{1} \sigma} S(\sigma) d \sigma$, one can obtain

$$
\phi_{1}(k \tau)=\int_{(k-1) \tau}^{k \tau} r e^{d_{1}(\sigma-k \tau)} S(\sigma) d \sigma=\int_{k \tau}^{(k+1) \tau} r e^{d_{1}(\sigma-(k+1) \tau)} S(\sigma-\tau) d \sigma .
$$

So we obtain $u((k+1) \tau)=0$ and so $S_{j}(\tau)>0$. Hence $S_{j}((k+1) \tau)>0$. Since $u(t)$ is strictly decreasing, we have $S_{j}(t)>u(t)>0$ for all $t>0$.

Let $R^{+}=[0, \infty), R_{+}^{5}=\left\{x \in R^{5}: x \geq 0, x=\left(S_{j}, S, I, y_{j}, y\right)\right\}$. Denote $f=\left(f_{1}, f_{2}, f_{3}, f_{4}, f_{5}\right)^{T}$ the map defined by the right-hand side of the anterior five equations of system (1.1), and $N$ the set of all non-negative integers. Let $V: R_{+} \times R_{+}^{5} \rightarrow R_{+}$, then $V$ is said to belong to class $V_{0}$ if

(i) $V$ is continuous in $((k-1) T, k T] \times R_{+}^{5}, k \in N$, and for each $x \in R_{+}^{5}$ limits

$$
\lim _{(t, z) \rightarrow\left((k-1) T^{+}, x\right)} V(t, z)=V((k-1) T, x), \quad \lim _{(t, z) \rightarrow\left(k T^{+}, x\right)} V(t, z)=V\left(k T^{+}, x\right)
$$

exist.

(ii) $V$ is locally Lipschitzian in $x$.

Then we have the following definition:

Definition 1. [3] Let $V \in V_{0}$, then for $(t, x) \in((k-1) T, k T] \times R_{+}^{5}, k \in N$, the upper right derivative of $V(t, x)$ with respect to the impulsive differential system (1.1) is defined as

$$
D^{+} V(t, x)=\limsup _{h \rightarrow 0^{+}} \frac{1}{h}[V(t+h, x+h f(t, x))-V(t, x)] .
$$

Next, we will consider the boundedness of system (1.1). Let

$$
V(t)=\lambda S_{j}(t)+\lambda S(t)+\lambda I(t)+y_{j}(t)+y(t) .
$$

Then the upper right derivative of $V(t)$ along a solution of system (1.1) with $t \neq(n+l-1) T, t \neq n T$ is given by

$$
\dot{V}(t)=r \lambda S(t)-d_{2} \lambda S^{2}(t)-d_{1} \lambda S_{j}(t)-d_{3} \lambda I(t)-d_{4} y_{j}(t)-d_{5} y(t) .
$$

Since $r, \lambda, d_{1}, d_{2}, d_{3}, d_{4}, d_{5}>0$, one can deduce that

$$
\dot{V}(t)<\lambda\left((r+d) S(t)-d_{2} S^{2}(t)\right)-d V(t)<\Lambda-d V(t),
$$


where $\Lambda=\lambda(r+d)^{2} /\left(4 d_{2}\right)$ and $d=\min \left\{d_{1}, d_{3}, d_{4}, d_{5}\right\}$. We consider the following impulse differential inequalities.

$$
\left\{\begin{array}{l}
\dot{V}(t) \leq \Lambda-d V(t), \quad t \neq(n+l-1) T, \quad t \neq n T \\
V\left((n+l-1) T^{+}\right)=V((n+l-1) T)+q, \quad t=(n+l-1) T \\
V\left(n T^{+}\right)=V(n T)+\lambda p, \quad t=n T .
\end{array}\right.
$$

According to impulse differential inequalities theory, we get

$$
\begin{aligned}
V(t) \leq & V(0) e^{-d t}+\int_{0}^{t} \Lambda e^{-d(t-s)} d s \\
& +\sum_{0<(n+l-1) T<n T<t}\left(q e^{-d(t-(n+l-1) T)}+\lambda p e^{-d(t-n T)}\right) \\
& \rightarrow \frac{\Lambda}{d}+\frac{q e^{d l T}+\lambda p e^{d T}}{e^{d T}-1}=L
\end{aligned}
$$

as $t \rightarrow \infty$, so $V(t)$ is ultimately bounded. Therefore, by the definition of $V(t)$, we know that each positive solution of system is ultimately bounded.

\section{Analysis of the Model}

Then we have the following lemmas from previous work.

Lemma 1. $[2,8]$ Let $V: R_{+} \times R_{+}^{3} \rightarrow R_{+}$, and $V \in V_{0}$. Assume that

$$
\begin{aligned}
D^{+} V(t, z(t)) & \leq(\geq) g(t, V(t, z(t))), \quad t \neq n \tau, \\
V\left(t, z(t)^{+}\right) & \leq(\geq) \Psi_{n}(V(t, z(t))), \quad t=n \tau,
\end{aligned}
$$

where $g: R_{+} \times R_{+} \rightarrow R$ is continuous in $(n \tau,(n+1) \tau] \times R_{+}$and for each $x \in R_{+}, n \in N, \lim _{(t, y) \rightarrow\left((n \tau)^{+}, x\right)} g(t, y)=g\left((n \tau)^{+}, x\right)$ exist; $\Psi_{n}: R_{+} \rightarrow R_{+}$is nondecreasing. Let $r(t)=r\left(t, 0, u_{0}\right)$ be the maximal (minimal) solution of the scalar impulsive differential equation

$$
\begin{cases}u^{\prime}=g(t, u), & t \neq n \tau \\ u\left(t^{+}\right)=\Psi_{n}(u(t)), & t=n \tau\end{cases}
$$

on $[0, \infty)$. Then $V\left(0^{+}, z_{0}\right) \leq(\geq) u_{0}$ implies that $V(t, z(t)) \leq(\geq) r(t), t \geq 0$, where $z(t)=z\left(t, 0, z_{0}\right)$ is a solution of system $(2.1)$ on $[0, \infty)$.

Lemma 2. [18] Consider the following delay differential equation

$$
\frac{d x(t)}{d t}=a x(t-\tau)-b x(t)-c x^{2}(t)
$$

where $a, b, c, \tau$ are all positive constants and $x(t)>0$ for $t \in[-\tau, 0]$. Then we have

(1) if $a<b, \lim _{t \rightarrow \infty} x(t)=0$. 
(2) if $a>b, \lim _{t \rightarrow \infty} x(t)=(a-b) / c$.

Lemma 3. [11] System

$$
\begin{cases}\frac{d v}{d t}=-c v(t), & t \neq n T, n \in N \\ \Delta v(t)=\mu, & t=n T, n \in N\end{cases}
$$

has a unique positive T-periodic solution $v^{*}(t)=\mu e^{-c(t-n T)} /\left(1-e^{-c T}\right)$ for $t \in(n T,(n+1) T], n \in N$ with $v^{*}(0)=\mu /\left(1-e^{-c T}\right)$. And for each solution $v(t)$ we have $v(t) \rightarrow v^{*}(t)$ as $t \rightarrow+\infty$.

Lemma 4. [22] System

$$
\left\{\begin{array}{l}
\frac{d u(t)}{d t}=a v(t)-b u(t), \\
\frac{d v(t)}{d t}=-c v(t), \\
\Delta u(t)=0, \quad \Delta v(t)=\mu, \quad t=n T
\end{array}\right\}
$$

has a unique positive T-periodic solution $\left(u^{*}(t), v^{*}(t)\right)$, which takes the form

$$
\left\{\begin{array}{l}
u^{*}(t)=u^{*}(0) e^{-b(t-n T)}+\frac{a \mu\left(e^{-b(t-n T)}-e^{-c(t-n T)}\right)}{(c-b)\left(1-e^{-c T}\right)}, \\
v^{*}(t)=\mu e^{-c(t-n T)} /\left(1-e^{-c T}\right) \\
u^{*}(0)=\frac{a \mu\left(e^{-b T}-e^{-c T}\right)}{(c-b)\left(1-e^{-b T}\right)\left(1-e^{-c T}\right)} \\
v^{*}(0)=\mu /\left(1-e^{-c T}\right)
\end{array}\right.
$$

for $t \in(n T,(n+1) T]$ and $n \in N$, and satisfies $u(t) \rightarrow u^{*}(t)$ and $v(t) \rightarrow v^{*}(t)$ as $t \rightarrow \infty$.

\subsection{Global attractivity of the 'mature pest-extinction' periodic so- lution}

Firstly, in this section, we investigate the pest-extinction solution of the system (1.3), in which the pest individual are entirely absent from the population permanently, i.e. $S(t)=0, t \geq 0$. In this case, system system (1.3) can be rewritten as follows:

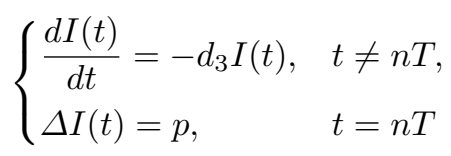

and

$$
\left\{\begin{array}{c}
\frac{d y_{j}(t)}{d t}=-\left(m+d_{4}\right) y_{j}(t), \\
\frac{d y(t)}{d t}=m y_{j}(t)-d_{5} y(t), \\
\Delta y_{j}(t)=q, \quad \Delta y(t)=0, \quad t=(n+l-1) T .
\end{array}\right.
$$


Then from Lemmas 3 and 4 we have

Theorem 1. System (1.3) has a 'pest-extinction' periodic solution $\left(0, I^{*}(t)\right.$, $\left.y_{j}^{*}(t), y^{*}(t)\right)$ for $t \in((n+l-1) T,(n+l) T], n \in N$, where $I^{*}(t)=\frac{p e^{-d_{3}(t-n T)}}{1-e^{-d_{3} T}}$, $I^{*}(0)=\frac{p}{1-e^{-d_{3} T}}$ and

$$
\left\{\begin{aligned}
y^{*}(t)= & y^{*}(0) e^{-d_{5}(t-(n+l-1) T)} \\
& +\frac{m q\left(e^{-d_{5}(t-(n+l-1) T)}-e^{-\left(m+d_{4}\right)(t-(n+l-1) T)}\right)}{\left(m+d_{4}-d_{5}\right)\left(1-e^{-\left(m+d_{4}\right) T}\right)} \\
y_{j}^{*}(t)= & q e^{-\left(m+d_{4}\right)(t-(n+l-1) T)} /\left(1-e^{-\left(m+d_{4}\right) T}\right) \\
y^{*}(0)= & \frac{m q\left(e^{-d_{5} T}-e^{-\left(m+d_{4}\right) T}\right)}{\left(m+d_{4}-d_{5}\right)\left(1-e^{-d_{5} T}\right)\left(1-e^{-\left(m+d_{4}\right) T}\right)} \\
y_{j}^{*}(0)= & q /\left(1-e^{-\left(m+d_{4}\right) T}\right)
\end{aligned}\right.
$$

Secondly, we analyze the global attractivity of the 'mature pest-extinction' periodic solution. Denote

$$
\begin{gathered}
A_{1}=\frac{p e^{-d_{3} T}}{1-e^{-d_{3} T}}, \quad A_{2}=\frac{m q\left(e^{-d_{5} T}-e^{-\left(m+d_{4}\right) T}\right)}{\left(m+d_{4}-d_{5}\right)\left(1-e^{-d_{5} T}\right)\left(1-e^{\left(m+d_{4}\right) T}\right)}, \\
B=\frac{m q\left(1-\frac{d_{5}}{m+d_{4}}\right)\left(\frac{d_{5}}{m+d_{4}}\left(1-e^{-\left(m+d_{4}\right) T}\right)\right)^{\frac{d_{5}}{m+d_{4}-d_{5}}}}{\left(m+d_{4}-d_{5}\right)\left(1-e^{-d_{5} T}\right)^{\frac{m+d_{4}}{m+d_{4}-d_{5}}}}, \quad \mathcal{R}_{1}=\frac{r e^{-d_{1} \tau}}{\beta A_{1}^{2}+\frac{\alpha A_{2}}{1+\omega A_{2}}} .
\end{gathered}
$$

Then we have

Theorem 2. The 'mature pest-extinction' periodic solution $\left(0, I^{*}(t), y_{j}^{*}(t)\right.$, $\left.y^{*}(t)\right)$ of system (1.3) is globally attractive, provided that $\mathcal{R}_{1}<1$.

Proof. Let $\left(S(t), I(t), y_{j}(t), y(t)\right)$ be any solution of system (1.3) with initial condition (1.2). From the second equation of system (1.3), it follows that $d I(t) / d t \geq-d_{3} I(t), I\left(t^{+}\right)=I(t)+p$, for $n T<t \leq(n+1) T$. By Lemma 3 , impulse differential system

$$
\begin{cases}\frac{d z_{1}(t)}{d t}=-d_{3} z_{1}(t), & t \neq n T, \\ \Delta z_{1}(t)=p, & t=n T\end{cases}
$$

has a globally asymptotically stable positive periodic solution,

$$
z_{1}^{*}(t)=\frac{p e^{-d_{3}(t-n T)}}{1-e^{-d_{3} T}}, \quad t \in(n T,(n+1) T] .
$$

Then the comparison theorem implies, for any sufficiently small $\varepsilon>0$, there exists an integer $n_{1}$ such that

$$
I(t)>z_{1}^{*}(t)-\varepsilon>\frac{p e^{-d_{3} T}}{1-e^{-d_{3} T}}-\varepsilon=A_{1}-\varepsilon, \quad t>n_{1} T .
$$


Similarly, for any sufficiently small $\varepsilon_{1}>0$, there exists an integer $n_{2}>n_{1}$ such that

$$
y_{j}(t)>y_{j}^{*}(t)-\varepsilon_{1}, \quad t \in((n+l-1) T,(n+l) T], t>n_{2} T,
$$

from which and the fourth equation of system (1.3), we have

$$
\frac{d y(t)}{d t} \geq m\left(y_{j}^{*}(t)-\varepsilon_{1}\right)-d_{4} y(t) .
$$

Consider the system

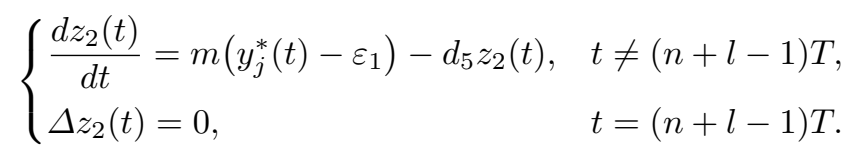

It is not difficult to verify that system (2.8) has a globally asymptotically stable positive periodic solution,

$$
z_{2}^{*}(t)=g(t)-m \varepsilon_{1} / d_{5}, \quad t \in((n+l-1) T,(n+l) T] .
$$

where $g(t)$ is continuous function on $[(n+l-1) T,(n+l) T]$ and

$$
g(t)=\frac{m q\left(\left(1-e^{-\left(m+d_{4} T\right)}\right) e^{-d_{5}(t-(n+l-1) T)}-\left(1-e^{-d_{5} T}\right) e^{-\left(m+d_{4}\right)(t-(n+l-1) T)}\right)}{\left(m+d_{4}-d_{5}\right)\left(1-e^{-\left(m+d_{4} T\right)}\right)\left(1-e^{-d_{5} T}\right)} .
$$

It has a unique stationary point

$$
\breve{t}=(n+l-1) T+\frac{1}{m+d_{4}-d_{5}} \ln \frac{\left(m+d_{4}\right)\left(1-e^{-d_{5} T}\right)}{d_{5}\left(1-e^{-\left(m+d_{4}\right) T}\right)},
$$

at which

$$
g^{\prime \prime}(\breve{t})=-m q \frac{d_{5}^{\frac{m+d_{4}}{m+d_{4}-d_{5}}}\left(1-e^{-\left(m+d_{4}\right) T}\right)^{\frac{d_{5}}{m+d_{4}-d_{5}}}}{\left(m+d_{4}\right)^{\frac{d_{5}}{m+d_{4}-d_{5}}}\left(1-e^{-d_{5} T}\right)^{\frac{m+d_{4}}{m+d_{4}-d_{5}}}}<0
$$

Note that

$g((n+l-1) T)=g((n+l) T)=\frac{m q\left(e^{-d_{5} T}-e^{-\left(m+d_{4}\right) T}\right)}{\left(m+d_{4}-d_{5}\right)\left(1-e^{-d_{5} T}\right)\left(1-e^{\left(m+d_{4}\right) T}\right)}=A_{2}$.

Thus we have

$$
A_{2} \leq g(t) \leq g(\breve{t})=B .
$$

So for any sufficiently small $\varepsilon_{2}>0$, there exists an integer $n_{2}>n_{1}$ and $n>n_{2}$ such that

$$
y(t)>z_{2}^{*}(t)-\varepsilon_{2}, \quad t \in((n+l-1) T,(n+l) T],
$$

which implies that

$$
y(t)>A_{2}-m \varepsilon_{1} / d_{5}-\varepsilon_{2}
$$


Therefore, from the first equation of system (1.3), we have

$$
\begin{aligned}
& \frac{d S(t)}{d t} \leq r e^{-d_{1} \tau} S(t-\tau)-d_{2} S^{2}(t) \\
& \quad-\left(\beta\left(A_{1}-\varepsilon\right)^{2}+\frac{\alpha\left(A_{2}-\frac{m \varepsilon_{1}}{d_{5}}-\varepsilon_{2}\right)}{1+\omega\left(A_{2}-\frac{m \varepsilon 1}{d_{5}}-\varepsilon_{2}\right)}\right) S(t), \quad t>n_{2} T+\tau
\end{aligned}
$$

Now we consider the following comparison equation:

$$
\begin{aligned}
& \frac{d z_{3}(t)}{d t}=r e^{-d_{1} \tau} z_{3}(t-\tau)-d_{2} z_{3}^{2}(t) \\
& \quad-\left(\beta\left(A_{1}-\varepsilon\right)^{2}+\frac{\alpha\left(A_{2}-\frac{m \varepsilon_{1}}{d_{5}}-\varepsilon_{2}\right)}{1+\omega\left(A_{2}-\frac{m \varepsilon_{1}}{d_{5}}-\varepsilon_{2}\right)}\right) z_{3}(t), \quad t>n_{2} T+\tau .
\end{aligned}
$$

Since $\mathcal{R}_{1}<1$, we have

$$
r e^{-d_{1} \tau}<\beta A_{1}^{2}+\alpha A_{2} /\left(1+\omega A_{2}\right) .
$$

We can choose three sufficiently small positive constants $\varepsilon, \varepsilon_{1}$, $\varepsilon_{2}$ such that

$$
r e^{-d_{1} \tau}<\beta\left(A_{1}-\varepsilon\right)^{2}+\frac{\alpha\left(A_{2}-\frac{m \varepsilon_{1}}{d_{5}}-\varepsilon_{2}\right)}{1+\omega\left(A_{2}-\frac{m \varepsilon_{1}}{d_{5}}-\varepsilon_{2}\right)} .
$$

By Lemma 2 we know $\lim _{t \rightarrow \infty} z_{3}(t)=0$. From $(2.11)$, by impulsive comparison theorem, we have $S(t) \leq z_{3}(t)$ for $t$ large enough. Then we obtain that $\lim _{t \rightarrow \infty} S(t)=0$. It implies, for a sufficiently small $\varepsilon_{3}>0$ and large $t$, we have $0<S(t)<\varepsilon_{3}$. Without loss of generality, we may assume $0<S(t)<\varepsilon_{3}$ as $t \geq 0$.

From the second equation of system (1.3), we have $d I(t) / d t<\left(\beta \varepsilon_{3} L-\right.$ $\left.d_{3}\right) I(t)$. Consider the following comparison system

$$
\begin{cases}\frac{d z_{4}(t)}{d t}=\left(\beta \varepsilon_{3} L-d_{3}\right) z_{4}(t), & t \neq n T, \\ \Delta z_{4}(t)=p, & t=n T .\end{cases}
$$

By Lemma 3, system (2.15) has a positive periodic solution

$$
z_{4}^{*}(t)=\frac{p e^{-\left(d_{3}-\beta \varepsilon L\right)(t-n T)}}{1-e^{-\left(d_{3}-\beta \varepsilon_{3} L\right) T}},
$$

which is globally asymptotically stable. Thus, for a sufficiently small $\varepsilon>0$, when $t$ is large enough, we have

$$
I(t)<z_{4}(t)<z_{4}^{*}(t)+\varepsilon .
$$

Combining system (2.6) with (2.16), we obtain

$$
z_{1}^{*}(t)-\varepsilon<I(t)<z_{4}^{*}(t)+\varepsilon .
$$

Let $\varepsilon_{1}, \varepsilon_{3} \rightarrow 0,(2.17)$ implies $\lim _{t \rightarrow \infty} I(t)=I^{*}(t)$. 
Consider the third equation of system (1.3), we have

$$
\frac{d y_{j}(t)}{d t} \leq \frac{\lambda \alpha \varepsilon L}{1+\omega L}-\left(m+d_{4}\right) y_{j}(t)
$$

From the comparison system

$$
\begin{cases}\frac{d z_{5}(t)}{d t}=\frac{\lambda \alpha \varepsilon_{3} L}{1+\omega L}-\left(m+d_{4}\right) z_{5}(t), & t \neq(n+l-1) T, \\ \Delta z_{5}(t)=q, & t=(n+l-1) T,\end{cases}
$$

there exists a positive periodic solution,

$$
z_{5}^{*}(t)=\frac{\lambda \alpha \varepsilon_{3} L}{(1+\omega L)\left(m+d_{4}\right)}+\frac{q e^{-\left(m+d_{4}\right)(t-(n+l-1) T)}}{1-e^{-\left(m+d_{4}\right) T}},(n+l-1) T<t \leq(n+l) T,
$$

which is globally asymptotically stable. Hence, for a sufficiently small $\varepsilon_{4}>0$, when $t$ is large enough, we have

$$
y_{j}(t)<z_{5}(t)<z_{5}^{*}(t)+\varepsilon_{4} .
$$

Combining system (2.7) with (2.18), we obtain

$$
y_{j}^{*}(t)-\varepsilon_{1}<y_{j}(t)<z_{5}^{*}(t)+\varepsilon_{4},
$$

which implies $\lim _{t \rightarrow \infty} y_{j}(t)=y_{j}^{*}(t)$ as $\varepsilon_{1}, \varepsilon_{3}$ and $\varepsilon_{4}$ are all sufficiently small constants. Since $\lim _{t \rightarrow \infty} y_{j}(t)=y_{j}^{*}(t)$, by Lemma 4 , we obtain $\lim _{t \rightarrow \infty} y(t)=$ $y^{*}(t)$. The proof is completed.

Let $\mathcal{R}_{1}=1$, we can work out threshold value of parameter $p, q$ and $\tau$ respectively. Denote

$$
p^{*}=\left(e^{d_{3} T}-1\right) \sqrt{\frac{r e^{-d_{1} \tau}-\frac{\alpha A_{2}}{1+\omega A_{2}}}{\beta}}, \quad \tau^{*}=\frac{1}{d_{1}} \ln \frac{r}{\beta A_{1}^{2}+\frac{\alpha A_{2}}{1+\omega A_{2}}}
$$

and

$$
q^{*}=\frac{\left(m+d_{4}-d_{5}\right)\left(1-e^{-d_{5} T}\right)\left(1-e^{\left(m+d_{4}\right) T}\right)\left(r e^{-d_{1} \tau}-\beta A_{1}^{2}\right)}{m\left(e^{-d_{5} T}-e^{-\left(m+d_{4}\right) T}\right)\left(\alpha-\omega\left(r e^{-d_{1} \tau}-\beta A_{1}^{2}\right)\right)} .
$$

We have

Corollary 1. If $p>p^{*}$ or $q>q^{*}$ or $\tau>\tau^{*}$, then the 'mature pest-extinction' periodic solution $\left(0, I^{*}(t), y_{j}^{*}(t), y^{*}(t)\right)$ is globally attractive.

\subsection{Permanence and the pest control strategy}

In Section 2.2, we will prove the pest-eradication solution $\left(0, I^{*}(t), y_{j}^{*}(t), y^{*}(t)\right)$ of (1.3) is globally attractive when $\mathcal{R}_{1}<1$, that is, the adult pest population is eradicated totally as time goes under the condition for the global attractivity. 
Considering the principle of ecosystem balance, biological diversity of population and resources saving, we hope that the pest population can coexist with its natural enemy population while the pests do not bring immense economic loss, in other words, we only want to control the pest population under the economic threshold level (ETL). So in the following, we will give the sufficient condition for the permanence of (1.3), and discuss the strategy of regulating the pest. The following definition is necessary before stating our theorems. Denote

$$
\begin{aligned}
A & =\frac{m \lambda \alpha L}{d_{4}(1+\omega L)\left(m+d_{4}\right)}, \quad \mathcal{R}_{2}=\left(r e^{-d_{1} \tau}-\frac{\beta p L}{1-e^{-d_{3} T}}\right) / \frac{\alpha B}{1+\omega B}, \\
B_{1} & =\omega B\left(\frac{\mathcal{R}_{2} \alpha A}{1+\omega B}-\left(d_{2}+\frac{\beta^{2} L^{3}}{d_{3}}\right)\right)-\left(d_{2}+\frac{\beta^{2} L^{3}}{d_{3}}+\alpha A\right), \\
k^{*} & =\frac{\mathcal{R}_{2}-1}{\mathcal{R}_{2}} \frac{B_{1}+\sqrt{B_{1}^{2}+4 \alpha \omega A B\left(d_{2}+\frac{\beta^{2} L^{3}}{d_{3}}\right)\left(\mathcal{R}_{2}-1\right)}}{2 \omega A\left(d_{2}+\frac{\beta^{2} L^{3}}{d_{3}}\right)} .
\end{aligned}
$$

Then we have

Theorem 3. If $\mathcal{R}_{2}>1$, there exists a positive constant $\sigma$ such that each positive solution $\left(S(t), I(t), y_{j}(t), y(t)\right)$ of system (1.3) satisfies $S(t) \geq \sigma$ for $t$ large enough.

Proof. The first equation of system (1.3) can be rewritten as

$$
\dot{S}(t)=\left(r e^{-d_{1} \tau}-d_{2} S(t)-\beta I^{2}(t)-\frac{\alpha y(t)}{1+\omega y(t)}\right) S(t)-r e^{-d_{1} \tau} \frac{d}{d t} \int_{t-\tau}^{t} S(\varrho) d \varrho .
$$

Let $U(t)=S(t)+r e^{-d_{1} \tau} \int_{t-\tau}^{t} S(\varrho) d \varrho$. Its derivative along the solution of system (1.3) is

$$
\frac{d U(t)}{d t}=\left(r e^{-d_{1} \tau}-d_{2} S(t)-\beta I^{2}(t)-\frac{\alpha y(t)}{1+\omega y(t)}\right) S(t) .
$$

Next, we claim that the inequality $S(t)<k^{*}$ cannot hold for all $t \geq t_{0}$, here $t_{0}>0$ is arbitrary constant. Otherwise, there exists a positive constant $t_{0}$ such that $S(t)<k^{*}$ for all $t \geq t_{0}$. We shall prove that this can not happen. From the second equation of system (1.3), when $t \geq t_{0}$, we have

$$
\begin{cases}\frac{d I(t)}{d t} \leq \beta k^{*} L^{2}-d_{3} I(t), & t \neq n T \\ \Delta I(t)=p, & t=n T .\end{cases}
$$

Thus there exists a $T_{1}^{\prime}>t_{0}+\tau$ such that

$$
I(t)<\frac{\beta k^{*} L^{2}}{d_{3}}+\frac{p e^{-d_{3}(t-n T)}}{1-e^{-d_{3} T}}+\varepsilon_{1}<\frac{\beta k^{*} L^{2}}{d_{3}}+\frac{p}{1-e^{-d_{3} T}}+\varepsilon_{1} \triangleq C, \quad t>T_{1}^{\prime} .
$$


From the third equation of system (1.3), when $t \geq t_{0}$, we have

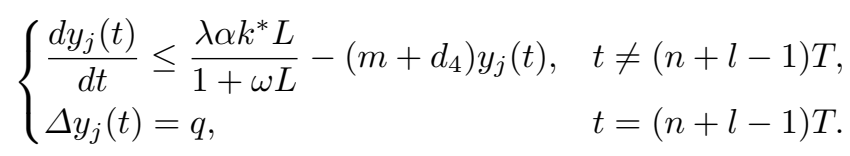

Thus there exists a $T_{1}^{\prime \prime}>t_{0}+\tau$ such that

$y_{j}(t)<\frac{\lambda \alpha k^{*} L}{(1+\omega L)\left(m+d_{4}\right)}+\frac{q e^{-\left(m+d_{4}\right)(t-(n+l-1) T)}}{1-e^{-\left(m+d_{4}\right) T}}+\varepsilon_{2}=\tilde{y}_{j}(t)+\varepsilon_{2}, \quad t>T_{1}^{\prime \prime}$.

Let $T_{1}=\max \left\{T_{1}^{\prime}, T_{1}^{\prime \prime}\right\}$. Then from the fourth equation of system (1.3), when $t>T_{1}$, we have

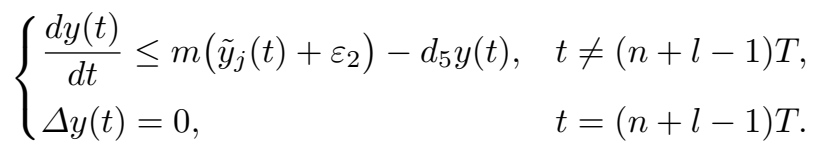

Similarly, there exits a $T_{2}>T_{1}$ such that

$$
y(t)<\frac{m}{d_{5}}\left(\frac{\lambda \alpha k^{*} L}{(1+\omega L)\left(m+d_{4}\right)}+\varepsilon_{2}\right)+g(t)+\varepsilon_{3}, \quad t>T_{2} .
$$

Then from (2.9), we have

$$
y(t)<\frac{m}{d_{5}}\left(\frac{\lambda \alpha k^{*} L}{(1+\omega L)\left(m+d_{4}\right)}+\varepsilon_{2}\right)+B+\varepsilon_{3} \triangleq D, \quad t>T_{2} .
$$

By the definition of $k^{*}$, one can easily get $k^{*}>0$. Choose $\varepsilon_{1}, \varepsilon_{2}, \varepsilon_{3}>0$ to be small enough such that

$$
r e^{-d_{1} \tau}>d_{2} k^{*}+\beta C L+\alpha D /(1+\omega D),
$$

inequality (2.23) is further proved in Appendix A. Then we have

$$
\frac{d U(t)}{d t}>\left(r e^{-d_{1} \tau}-d_{2} k^{*}-\beta C L-\frac{\alpha D}{1+\omega D}\right) S(t), \quad t>\tilde{T} .
$$

Let $S^{l}=\min _{t \in\left[T_{1}, T_{1}+\tau\right]} S(t)$. We can show $S(t) \geq S^{l}$ for all $t \geq \tilde{T}$. Actually, if there exists a nonnegative constant $T_{3}$ such that $S(t) \geq S^{l}$ for $t \in[\tilde{T}, \tilde{T}+\tau+$ $\left.T_{3}\right], S\left(\tilde{T}+\tau+T_{3}\right)=S^{l}$ and $\dot{S}\left(\tilde{T}+\tau+T_{3}\right) \leq 0$. Then from the first equation of (1.3) and (2.21), we easily see that

$$
\begin{aligned}
& \dot{S}\left(\tilde{T}+\tau+T_{3}\right)=r e^{-d_{1} \tau} S\left(\tilde{T}+T_{3}\right)-d_{2} S^{2}\left(\tilde{T}+\tau+T_{3}\right) \\
& \quad-\beta S\left(\tilde{T}+\tau+T_{3}\right) I^{2}\left(\tilde{T}+\tau+T_{3}\right)-\frac{\alpha S\left(\tilde{T}+\tau+T_{3}\right) y\left(\tilde{T}+\tau+T_{3}\right)}{1+\omega y\left(\tilde{T}+\tau+T_{3}\right)}
\end{aligned}
$$

So we have

$$
\dot{S}\left(\tilde{T}+\tau+T_{3}\right)>\left(r e^{-d_{1} \tau}-d_{2} k^{*}-\beta C L-\alpha D /(1+\omega D)\right) S^{l}>0,
$$


which is a contradiction. Hence $S(t) \geq S^{l}>0$ for all $t \geq \tilde{T}$. From (2.24), we have

$$
\frac{d U(t)}{d t}>\left(r e^{-d_{1} \tau}-d_{2} k^{*}-\beta C L-\frac{\alpha D}{1+\omega D}\right) S^{l}>0,
$$

which implies $U(t) \rightarrow+\infty$ as $t \rightarrow+\infty$. This is a contradiction to $U(t) \leq$ $\left(1+r \tau e^{-d_{1} \tau}\right) L$. Therefore, for any positive constant $t_{0}$, the inequality $S(t)<k^{*}$ cannot hold for all $t \geq t_{0}$.

If $S(t) \geq k^{*}$ holds true for all $t$ large enough, then we finish the proof of the theorem. Otherwise, $S(t)$ is oscillatory about $k^{*}$. We shall show our conclusion is also true in this case.

Let $\sigma=\min \left\{\frac{1}{2} k^{*}, k^{*} \exp \left(-\left(d_{2} L+\beta L^{2}+\frac{\alpha L}{1+\omega L}\right) \tau\right)\right\}$. In the following, we shall prove that $S(t) \geq \sigma$. Assume there exist two positive constants $\bar{t}, \rho$ such that

$$
S(\bar{t})=S(\bar{t}+\rho)=k^{*}, \quad \text { and } S(t)<k^{*}, \quad \text { for } \bar{t}<t<\bar{t}+\rho .
$$

From (2.27), we know that $S(t)<k^{*}$ for $\bar{t}<t<\bar{t}+\rho$, from the third equation of system (1.3), when $t \geq \bar{t}$, we have

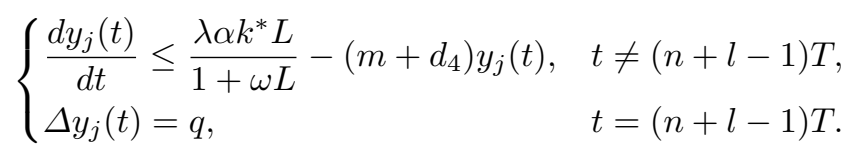

Thus there exists a $T_{1}>\bar{t}+\tau$ such that

$y_{j}(t)<\frac{\lambda \alpha k^{*} L}{(1+\omega L)\left(m+d_{4}\right)}+\frac{q e^{-\left(m+d_{4}\right)(t-(n+l-1) T)}}{1-e^{-\left(m+d_{4}\right) T}}+\varepsilon_{2}=\tilde{y}_{j}(t)+\varepsilon_{2}, \quad t>T_{1}$.

Then from the fourth equation of system (1.3), when $t>T_{1}$, we have

$$
\left\{\begin{array}{llrl}
\frac{d y(t)}{d t} \leq m\left(\tilde{y}_{j}(t)+\varepsilon_{2}\right)-d_{5} y(t), & & t \neq(n+l-1) T \\
\Delta y(t) & =0, & & t=(n+l-1) T
\end{array}\right.
$$

Similarly, there exits a $T_{2}>T_{1}$ such that

$$
\begin{aligned}
y(t) & <\frac{m}{d_{5}}\left(\frac{\lambda \alpha k^{*} L}{(1+\omega L)\left(m+d_{4}\right)}+\varepsilon_{2}\right)+g(t)+\varepsilon_{3} \\
& <\frac{m}{d_{5}}\left(\frac{\lambda \alpha k^{*} L}{(1+\omega L)\left(m+d_{4}\right)}+\varepsilon_{2}\right)+B+\varepsilon_{3}=D .
\end{aligned}
$$

Thus, we can eventually get $y(t)<D$ for $\bar{t}+T_{2}<t<\bar{t}+\rho$.

Since $S(t)$ is continuous and bounded, and is not effected by impulses, we conclude that $S(t)$ is uniformly continuous. Hence there exists a constant $T_{4}$ with $0<T_{4}<\tau$ and independent of the choice of $\bar{t}$ such that $S(t)>\frac{1}{2} k^{*}$ for all $\bar{t} \leq t \leq \bar{t}+T_{4}$. If $\rho \leq T_{4}$, our aim is obtained.

If $T_{4}<\rho \leq \tau$, from the first equation of system (1.3), we have

$$
\dot{S}(t) \geq-\left(d_{2} L+\beta L^{2}+\alpha L /(1+\omega L)\right) S(t), \quad \bar{t}<t \leq \bar{t}+\rho .
$$


Then we have $S(t) \geq k^{*} e^{-\left(d_{2} L+\beta L^{2}+\frac{\alpha L}{1+\omega L}\right) \tau}$ for $\bar{t}<t \leq \bar{t}+\rho \leq \bar{t}+\tau$ since $S(\bar{t})=k^{*}$. It is obvious that $S(t) \geq \sigma$ for $\bar{t}<t \leq \bar{t}+\rho$.

If $\rho \geq \tau$, then we have that $S(t) \geq k^{*} e^{-\left(d_{2} L+\beta L^{2}+\frac{\alpha L}{1+\omega L}\right) \tau}$ for $\bar{t}<t \leq$ $\bar{t}+\tau$. Next, we will show that $S(t) \geq k^{*} e^{-\left(d_{2} L+\beta L^{2}+\frac{\alpha L}{1+\omega L}\right) \tau}$ for $\bar{t}+\tau<t \leq$ $\bar{t}+\rho$. In fact, if this is not true, there exists a $T_{5}>0$ such that $S(t) \geq$ $k^{*} e^{-\left(d_{2} L+\beta L^{2}+\frac{\alpha L}{1+\omega L}\right) \tau}$ for $\bar{t}<t \leq \bar{t}+\tau+T_{5} ; S\left(\bar{t}+\tau+T_{5}\right)=k^{*} e^{-\left(d_{2} L+\beta L^{2}+\frac{\alpha L}{1+\omega L}\right) \tau}$ and $\dot{S}\left(\bar{t}+\tau+T_{5}\right) \leq 0$.

On the other hand, from the second equation of system (1.3) and (2.21), we easily see

$$
\begin{aligned}
& \dot{S}\left(\tilde{T}+\tau+T_{5}\right)=r e^{-d_{1} \tau} S\left(\tilde{T}+T_{5}\right)-d_{2} S^{2}\left(\tilde{T}+\tau+T_{5}\right) \\
& \quad-\beta S\left(\tilde{T}+\tau+T_{5}\right) I^{2}\left(\tilde{T}+\tau+T_{5}\right)-\frac{\alpha S\left(\tilde{T}+\tau+T_{5}\right) y\left(\tilde{T}+\tau+T_{5}\right)}{1+\omega y\left(\tilde{T}+\tau+T_{5}\right)},
\end{aligned}
$$

thus we get

$$
\dot{S}\left(\tilde{T}+\tau+T_{5}\right)>\left(r e^{-d_{1} \tau}-d_{2} k^{*}-\beta C L-\frac{\alpha D}{1+\omega D}\right) S^{l}>0,
$$

which is a contradiction to $\dot{S}\left(\bar{t}+\tau+T_{5}\right) \leq 0$. Hence we get that $S(t) \geq \sigma>0$ for all $t \in[\bar{t}, \bar{t}+\rho]$. Since interval $[\bar{t}, \bar{t}+\rho]$ is arbitrarily chosen, we know $S(t) \geq \sigma$ for $t$ large enough. Please notice the choice of $\sigma$ is independent of the positive solution of (1.3) which satisfies that $I(t) \geq \sigma$ for sufficiently large $t$. This completes the proof.

Theorem 4. If $\mathcal{R}_{2}>1$, then system (1.3) is permanent.

Proof. Suppose that $\left(S(t), I(t), y_{j}(t), y(t)\right)$ is any positive solution of system (1.3) with initial conditions (1.2). By Theorem 3, there exist positive constants $\sigma$ and $T^{*}$ such that $S(t) \geq \sigma$, for $t \geq T^{*}$. From the proof of Theorem 2, we can conclude that the following inequalities

$$
\begin{aligned}
& I(t)>A_{1}-\varepsilon_{1}, \quad y_{j}(t)>y_{j}^{*}(t)-\varepsilon_{1}>\frac{q e^{-\left(m+d_{4}\right) T}}{1-e^{-\left(m+d_{4}\right) T}}-\varepsilon_{1}, \\
& y(t)>A_{2}-m \varepsilon_{1} / d_{5}-\varepsilon_{2}
\end{aligned}
$$

hold for $t$ large enough. Noticing the boundedness of (1.1) gives $S(t), I(t), y_{j}(t)$, $y(t) \leq L$ for $t$ large enough. Then system (1.3) is permanent, and the proof is completed.

Let $\mathcal{R}_{2}=1$, we can work out threshold value of parameter $p, q$ and $\tau$ respectively. Denote

$$
p_{*}=\frac{\left(1-e^{-d_{3} T}\right)\left(r e^{-d_{1} \tau}-\frac{\alpha B}{1+\omega B}\right)}{\beta L}, \quad \tau_{*}=\frac{1}{d_{1}} \ln \frac{r}{\frac{\beta p L}{1-e^{-d_{3} T}}+\frac{\alpha B}{1+\omega B}}
$$

and

$$
q_{*}=\frac{\left(m+d_{4}-d_{5}\right)\left(1-e^{-d_{5} T}\right)^{\frac{m+d_{4}}{m+d_{4}-d_{5}}}\left(\frac{r e^{-d_{1} \tau}-\beta p L /\left(1-e^{-d_{3} T}\right)}{\alpha-\omega\left(r e^{-d_{1} \tau}-\beta p L /\left(1-e^{-d_{3} T}\right)\right)}\right)}{m\left(1-\frac{d_{5}}{m+d_{4}}\right)\left(\frac{d_{5}}{m+d_{4}}\left(1-e^{-\left(m+d_{4}\right) T}\right)\right)^{\frac{d_{5}}{m+d_{4}-d_{5}}}} .
$$


We have

Corollary 2. If $p<p_{*}$ or $q<q_{*}$ or $\tau<\tau_{*}$, then system (1.3) is permanent.

As any pest control professional can tell us, large-scale eradication is infeasible. Therefore we aim to keep pests under the economic threshold level (ETL) to protect the crop but to eradicate them. In the following, we consider pest control strategy and give conditions under which the pest population is under ETL. Then we have

Theorem 5. For system (1.3), if

$$
r e^{-d_{1} \tau}-d_{2} E<\beta A_{1}^{2}+\alpha A_{2} /\left(1+\omega A_{2}\right)<r e^{-d_{1} \tau},
$$

then the pest and its natural enemy may coexist. Furthermore, when $t$ is large enough, we have $S(t)<E$, where the constant $E$ is the economic threshold level $(E T L)$.

Proof. Suppose $\left(S(t), I(t), y_{j}(t), y(t)\right)$ is a positive solution of (1.3) with initial conditions (1.2). Since

$$
\beta A_{1}^{2}+\alpha A_{2} /\left(1+\omega A_{2}\right)<r e^{-d_{1} \tau},
$$

we may choose three sufficiently small positive constants $\varepsilon_{1}, \varepsilon_{2}$, $\varepsilon$ such that

$$
r e^{-d_{1} \tau}>\beta\left(A_{1}-\varepsilon\right)^{2}+\frac{\alpha\left(A_{2}-m \varepsilon_{1} / d_{5}-\varepsilon_{2}\right)}{1+\omega\left(A_{2}-m \varepsilon_{1} / d_{5}-\varepsilon_{2}\right)} .
$$

Furthermore, from the following inequality

$$
r e^{-d_{1} \tau}-d_{2} E<\beta A_{1}^{2}+\alpha A_{2} /\left(1+\omega A_{2}\right)
$$

we can get

$$
\frac{r e^{-d_{1} \tau}-\left(\beta\left(A_{1}-\varepsilon\right)^{2}+\frac{\alpha\left(A_{2}-m \varepsilon_{1} / d_{5}-\varepsilon_{2}\right)}{1+\omega\left(A_{2}-m \varepsilon_{1} / d_{5}-\varepsilon_{2}\right)}\right)}{d_{2}}<E,
$$

and by (2.11), when $t$ is large enough, we have

$$
\frac{d S(t)}{d t} \leq r e^{-d_{1} \tau} S(t-\tau)-d_{2} S^{2}(t)-\left(\beta\left(A_{1}-\varepsilon\right)^{2}+\frac{\alpha\left(A_{2}-\frac{m \varepsilon_{1}}{d_{5}}-\varepsilon_{2}\right)}{1+\omega\left(A_{2}-\frac{m \varepsilon_{1}}{d_{5}}-\varepsilon_{2}\right)}\right) S(t),
$$

which implies $S(t) \leq Z(t)$ for $t$ large enough.

Consider the comparison equation

$\frac{d Z(t)}{d t}=r e^{-d_{1} \tau} Z(t-\tau)-d_{2} Z^{2}(t)-\left(\beta\left(A_{1}-\varepsilon\right)^{2}+\frac{\alpha\left(A_{2}-m \varepsilon_{1} / d_{5}-\varepsilon_{2}\right)}{1+\omega\left(A_{2}-m \varepsilon_{1} / d_{5}-\varepsilon_{2}\right)}\right) Z(t)$.

Using (2.28) and Lemma 2, we have

$$
\lim _{t \rightarrow+\infty} Z(t)=\frac{r e^{-d_{1} \tau}-\left(\beta\left(A_{1}-\varepsilon\right)^{2}+\frac{\alpha\left(A_{2}-m \varepsilon_{1} / d_{5}-\varepsilon_{2}\right)}{1+\omega\left(A_{2}-m \varepsilon_{1} / d_{5}-\varepsilon_{2}\right)}\right)}{d_{2}}
$$



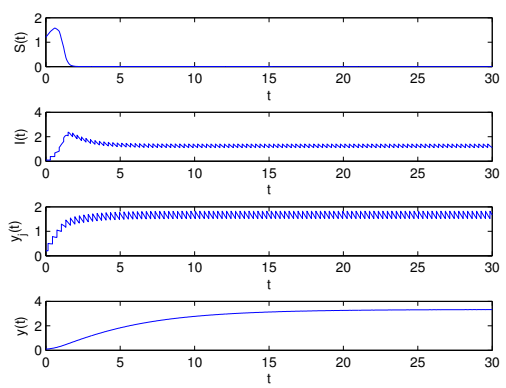

(a) Time series of system (1.3). Mature pests die out.

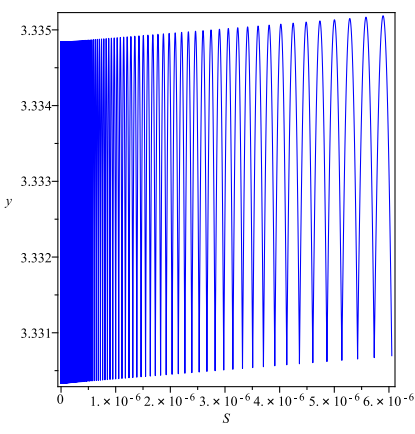

(b) Phase portrait of $S(t), y(t)$.

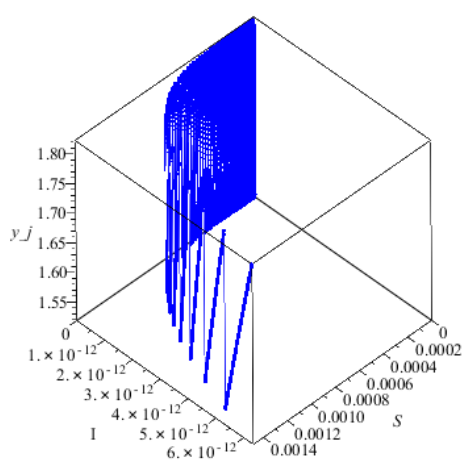

(c) Phase portrait of $S(t), I(t), y_{j}(t)$.

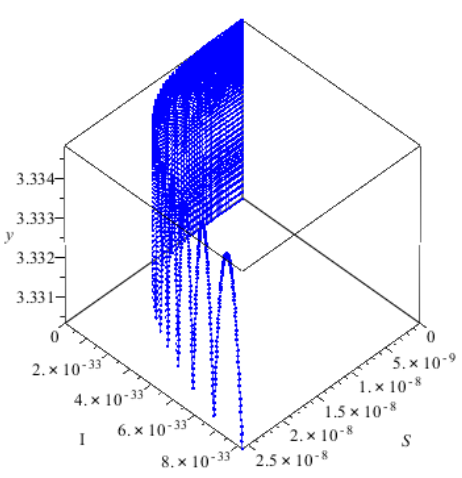

(d) Phase portrait of $S(t), I(t), y(t)$.

Figure 1. The results of numerical simulation on the threshold values $\mathcal{R}_{1}=0.3608$, where $l=0.5 ; p=0.3 ; q=0.3, T=0.3 ; \tau=0$.

which along with (2.30) gives

$$
\limsup _{t \rightarrow+\infty} S(t) \leq \frac{r e^{-d_{1} \tau}-\left(\beta\left(A_{1}-\varepsilon\right)^{2}+\frac{\alpha\left(A_{2}-m \varepsilon_{1} / d_{5}-\varepsilon_{2}\right)}{1+\omega\left(A_{2}-m \varepsilon_{1} / d_{5}-\varepsilon_{2}\right)}\right)}{d_{2}}<E .
$$

This completes the proof.

\section{Numerical Analysis and Discussion}

In this paper, we further developed impulsive delayed models with staged structure, and investigated a high-dimensional delayed pest management SI model with impulsive natural enemies and diseased pest transmission at different fixed moments. Our main purpose is to study dynamics of the model such as attractivity of periodic solution, permanence of the system and to give pest control strategies for Integrated Pest Management (IPM). Using the theory for impulsive delay differential equation, we obtained some interesting results. In 

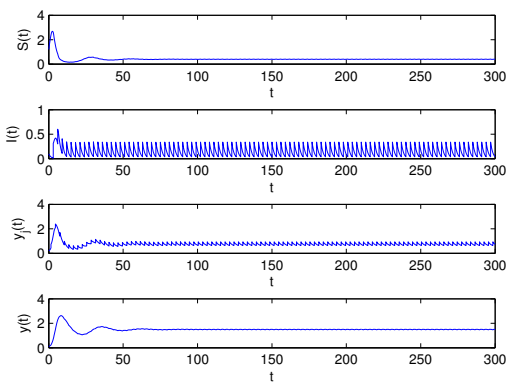

(a) Time series of system (1.3). System (1.3) is uniformly permanent.

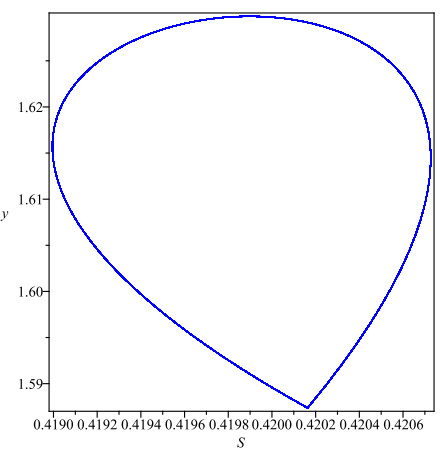

(b) Phase portrait of $S(t), y(t)$.

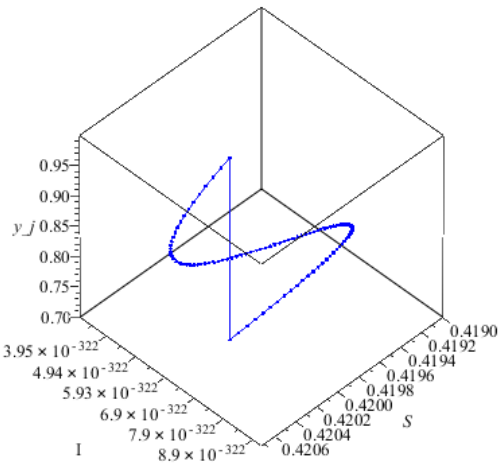

(c) Phase portrait of $S(t), I(t), y_{j}(t)$.

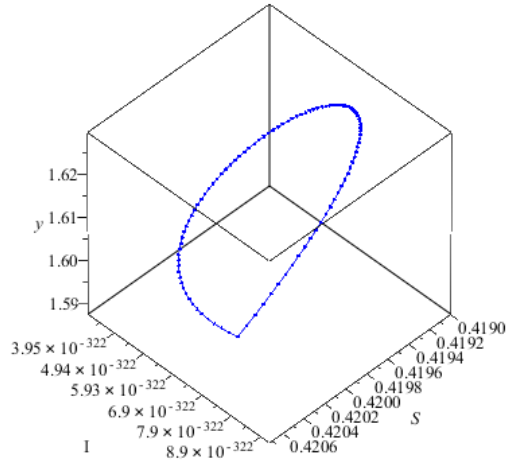

(d) Phase portrait of $S(t), I(t), y(t)$.

Figure 2. The results of numerical simulation on the threshold values $\mathcal{R}_{2}=1.6742$, where $l=0.5 ; p=0.3 ; q=0.3, T=3 ; \tau=0$.

Section 2, we analyzed extinction of pests and coexistence of pests and natural enemies. Section 2.1, discussed the conditions for the global asymptotical attractivity of the 'pest-extinction' periodic solution, and in Section 2.2, we got the conditions for the permanence of the system and also considered the pest control strategy. From Theorem 2, we can see that a large amount of infective prey input, $p$ or a large amount of natural enemy input, $q$ or a long juvenile period of the predator, $\tau$ is a sufficient condition for the global attractivity of the 'pest-extinction' periodic solution. From Theorems 3 and 4, we can see that a small amount of infective pest or a small amount of natural enemy or a short juvenile period of the predator (with $\tau$ ) is a sufficient condition for the permanence of the system. Theorems 2,3 and 4 show that $\mathcal{R}_{1}$ and $\mathcal{R}_{2}$ depend on the time delay $\tau$, so, we call it "profitless", and we obtained critical values of time delay $\tau^{*}$ and $\tau_{*}$.

To verify the theoretical results obtained in this paper, we will give some numerical simulations by Maple and Matlab, which also show some new phenomena different to previous work. For this purpose, parameters have been 

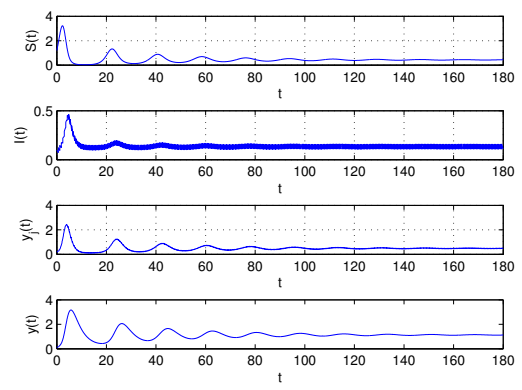

(a) Time series of system (1.3). System (1.3) is uniformly permanent.

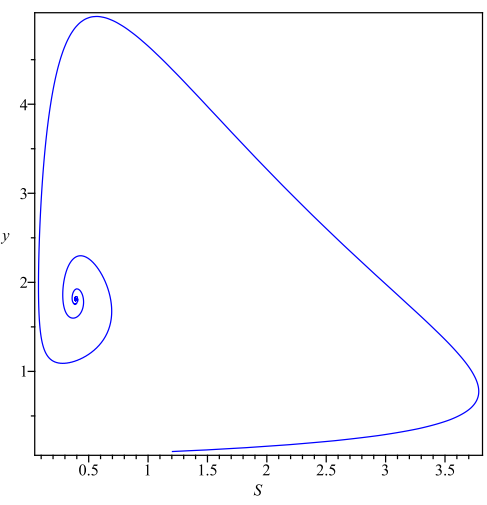

(b) Phase portrait of $S(t), y(t)$.

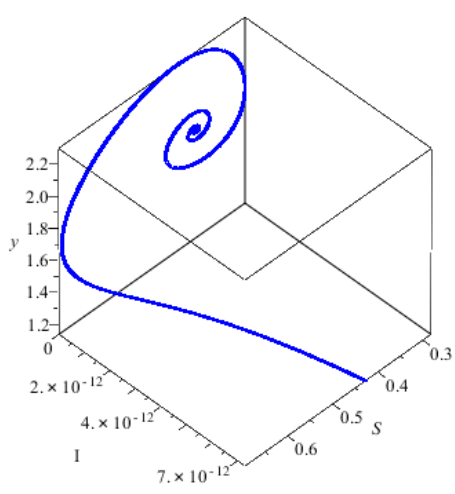

(d) Phase portrait of $S(t), I(t), y(t)$.

(c) Phase portrait of $S(t), I(t), y_{j}(t)$.

Figure 3. The results of numerical simulation on the threshold values $\mathcal{R}_{2}=7.1749$, where $l=0.5 ; p=0.05 ; q=0.05, T=0.3 ; \tau=0$.

selected as follows, $r=1, d_{1}=0.2, d_{2}=0.2, d_{3}=0.8, d_{4}=0.2, d_{5}=0.2$, $\beta=1.3, \alpha=1.3, \lambda=1, \omega=0.8, m=0.4$ with initial values $S(0)=1.2$, $I(0)=0.1, y_{j}(0)=0.2, y(0)=0.1$.

(i) From Theorem 3.2, we know that the mature pest tend to die out when $T=0.3, p=0.3, q=0.3, \tau=0\left(\mathcal{R}_{1}=0.3608<1\right.$, see Fig. $\left.1(\mathrm{a})\right)$.

(ii) If we increase the period of pulses to $T=3\left(\mathcal{R}_{2}=1.6742>1\right)$ or we fix $T=0.3$ and decrease the natural enemy amount and diseased pest amount to $p=0.05, q=0.05\left(\mathcal{R}_{2}=7.1749>1\right)$, then the system (1.3) is permanent (see Fig. 2(a) and Fig. 3(a), respectively), which follows from Theorem 4.

(iii) Also we can fix $T=3, p=0.3, q=0.3$ and increase maturation time delay to $\tau=5.8\left(\mathcal{R}_{1}=0.9787<1\right)$, then we find that the pest tends to be extinct (see Fig. 4), which implies the great effect of the maturation time delay on dynamics of the system.

(iv) Fig. 2(b), Fig. 2(c) and Fig. 2(d) show the system has a global stable 

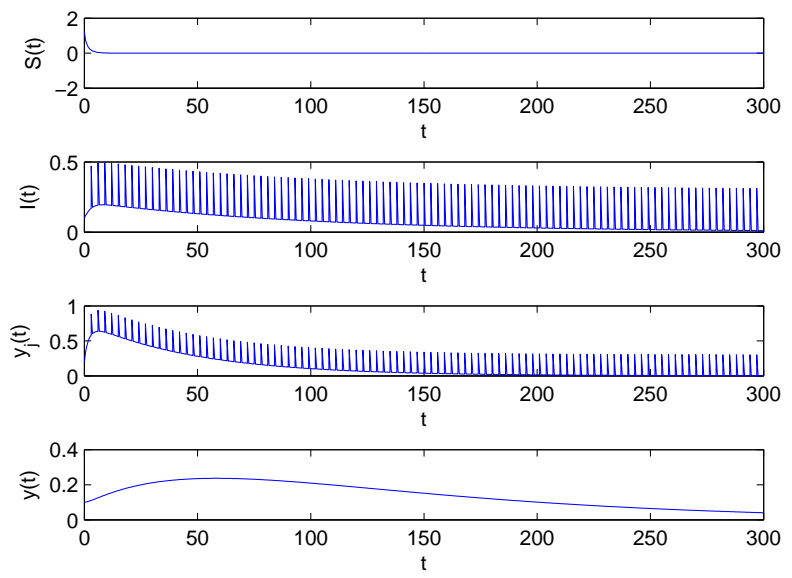

Figure 4. Time series of system (1.3).

$l=0.5 ; p=0.3 ; q=0.3, T=3 ; \tau=1$ and $\mathcal{R}_{1}=0.9787$.

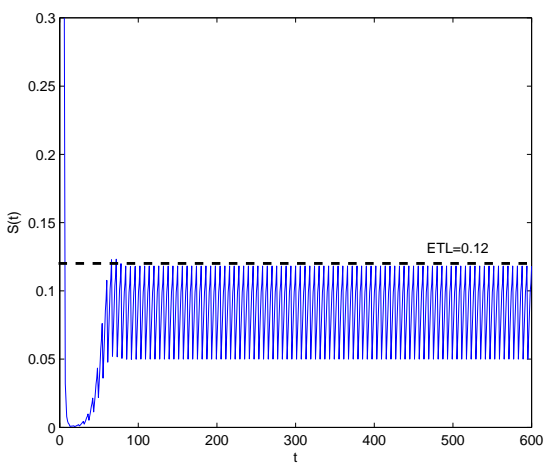

Figure 5. Time series of pests. Pests can be controlled under ETL.

positive periodic solution when it is permanent.

(v) From the standpoint of ecological balance and saving resources, we maintain the pest population under the economic threshold level $(\mathrm{ETL}=0.12)$ only, but to eradicate the pests totally (see Fig. 5).

All these results show that dynamical behaviors of system (1.3) become more complex under periodically impulsive effects.

\section{Appendix A}

The proof of inequality (2.23). Let

$$
r e^{-d_{1} \tau}>d_{2} k^{* *}+\beta C L+\alpha D /(1+\omega D) .
$$


From (2.21) and (2.22), and let $\varepsilon_{1}, \varepsilon_{2}, \varepsilon_{3} \rightarrow 0$, then we have

$$
r e^{-d_{1} \tau}>d_{2} k^{* *}+\beta\left(\frac{\beta k^{* *} L^{2}}{d_{3}}+\frac{p}{1-e^{-d_{3} T}}\right) L+\frac{\alpha\left(A k^{* *}+B\right)}{1+\omega\left(A k^{* *}+B\right)} .
$$

Simplifying the inequality $(2.23)$ we get

$$
\begin{aligned}
& \omega A\left(d_{2}+\frac{\beta^{2} L^{3}}{d_{3}}\right)\left(k^{* *}\right)^{2}+\left(\frac{p L}{1-e^{-d_{3} T}}-r e^{-d_{1} \tau}\right)(1+\omega B)+\alpha B \\
& \quad+\left((1+\omega B)\left(d_{2}+\frac{\beta^{2} L^{3}}{d_{3}}\right)+\left(\frac{p L}{1-e^{-d_{3} T}}-r e^{-d_{1} \tau}\right) A \omega+\alpha A\right) k^{* *}<0 .
\end{aligned}
$$

Note that

$$
\begin{aligned}
& r e^{-d_{1} \tau}-\frac{\beta p L}{1-e^{-d_{3} T}}=\mathcal{R}_{2} \frac{\alpha B}{1+\omega B} \\
& B_{1}=\omega B\left(\frac{\mathcal{R}_{2} \alpha A}{1+\omega B}-\left(d_{2}+\frac{\beta^{2} L^{3}}{d_{3}}\right)\right)-\left(d_{2}+\frac{\beta^{2} L^{3}}{d_{3}}+\alpha A\right) .
\end{aligned}
$$

Then we have

$$
\omega A\left(d_{2}+\frac{\beta^{2} L^{3}}{d_{3}}\right)\left(k^{* *}\right)^{2}-B_{1} k^{* *}+\alpha B\left(1-\mathcal{R}_{2}\right)<0 .
$$

Solving the inequality (3.2), we get $k_{1}^{* *}<k^{* *}<k_{2}^{* *}$, where

$$
k_{1,2}^{* *}=\frac{B_{1} \mp \sqrt{B_{1}^{2}+4 \omega \alpha A B\left(d_{2}+\beta^{2} L^{3} / d_{3}\right)\left(\mathcal{R}_{2}-1\right)}}{2 \omega A\left(d_{2}+\beta^{2} L^{3} / d_{3}\right)} .
$$

In fact, inequality (3.1) holds for any $k^{* *}\left(k_{1}^{* *}<k^{* *}<k_{2}^{* *}\right)$. Thus, let

$$
0<k^{*}=\frac{\mathcal{R}_{2}-1}{\mathcal{R}_{2}} k_{2}^{* *}<k_{2}^{* *},
$$

then inequality (2.23) holds for $\mathcal{R}_{2}>1$.

\section{Acknowledgments}

We thank the referees and the editor for their careful reading of the original manuscript and many valuable comments and suggestions that greatly improved the presentation of this paper. T.Q. Zhang is financially supported by a Project of Shandong Province Higher Educational Science and Technology Program (No. J13LI05). X.Z. Meng is financially supported by the Shandong Provincial Natural Science Foundation, China (No. ZR2012AM012) and the SDUST Research Fund (No. 2011KYTD105).

\section{References}

[1] W.G. Aiello and H.I. Freedman. A time-delay model of single-species growth with stage structure. Math. Biosci., 101(2):139-153, 1990.

http://dx.doi.org/10.1016/0025-5564(90)90019-U. 
[2] D. Bainov and P. Simeonov. System with Impulsive Effect: Stability, Theory and Applications. John Wiley and Sons, New York, 1989.

[3] K.L. Cooke. Stability analysis for a vector disease model. Rocky Mountain J. Math., 9(1):31-42, 1979. http://dx.doi.org/10.1216/RMJ-1979-9-1-31.

[4] J. Cui and X. Song. Permanence of a predator-prey system with stage structure. Discrete Contin. Dyn. Syst. Ser. B, 4(3):547-554, 2004.

http://dx.doi.org/10.3934/dcdsb.2004.4.547.

[5] S. Gao, L. Chen, J.J. Nieto and A. Torres. Analysis of a delayed epidemic model with pulse vaccination and saturation incidence. Vaccine, 24(35):6037-6045, 2009 .

[6] S.A. Gourley and Y. Kuang. A stage structured predator-prey model and its dependence on maturation delay and death rate. J. Math. Biol., 49(2):188-200, 2004. http://dx.doi.org/10.1007/s00285-004-0278-2.

[7] J. Hui and D. Zhu. Dynamic complexities for prey-dependent consumption integrated pest management models with impulsive effects. Chaos Solitons Fractals, 29(1):233-251, 2006. http://dx.doi.org/10.1016/j.chaos.2005.08.025.

[8] V. Lakshmikantham, D. Bainov and P. Simeonov. Theory of Impulsive Differential Equations. World Scientific, Singapore, 1989.

[9] B. Leonid and B. Elena. Linearized oscillation theory for a nonlinear delay impulsive equation. J. Comput. Appl. Math., 161(2):477-495, 2003. http://dx.doi.org/10.1016/j.cam.2003.06.004.

[10] Z. Li and L. Chen. Periodic solution of a turbidostat model with impulsive state feedback control. Nonlinear Dynam., 58(3):525-538, 2009.

http://dx.doi.org/10.1007/s11071-009-9498-8.

[11] B. Liu, L. Chen and Y. Zhang. The effects of impulsive toxicant input on a population in a polluted environment. J. Biol. Syst., 11(3):265-274, 2003. http://dx.doi.org/10.1142/S0218339003000907.

[12] W. Liu, H.W. Hethcote and S.A. Levin. Dynamical behavior of epidemiological models with nonlinear incidence rates. J. Math. Biol., 25(4):359-380, 1986. http://dx.doi.org/10.1007/BF00277162.

[13] X. Liu and G. Ballinger. Boundedness for impulsive delay differential equations and applications to population growth models. Nonlinear Anal., 53(7):10411062, 2003. http://dx.doi.org/10.1016/S0362-546X(03)00041-5.

[14] X. Meng and L. Chen. A stage-structured SI eco-epidemiological model with time delay and impulsive controlling. J. Syst. Sci. Complex., 21(3):427-440, 2008. http://dx.doi.org/10.1007/s11424-008-9124-8.

[15] J.J. Nieto and R. Rodriguez-Lopez. New comparison results for impulsive integro-differential equations and applications. J. Math. Anal. Appl., 328(2):1343-1368, 2007. http://dx.doi.org/10.1016/j.jmaa.2006.06.029.

[16] Y. Pei, C. Li and L. Chen. Continuous and impulsive harvesting strategies in a stage-structured predator-prey model with time delay. Math. Comput. Simulation, 79(10):2994-3008, 2009. http://dx.doi.org/10.1016/j.matcom.2009.01.003.

[17] R. Shi and L. Chen. The study of a ratio-dependent predator-prey model with stage structure in the prey. Non. Dyn., 58(1):443-451, 2009.

http://dx.doi.org/10.1007/s11071-009-9491-2. 
[18] X. Song and L. Chen. Optimal harvesting and stability for a two-species competitive system with stage structure. Math. Biosci., 170(2):173-186, 2001. http://dx.doi.org/10.1016/S0025-5564(00)00068-7.

[19] S. Tang and L. Chen. Density-dependent birth rate, birth pulses and their population dynamic consequences. J. Math. Biol., 44(2):185-199, 2002. http://dx.doi.org/10.1007/s002850100121.

[20] W. Wang and L. Chen. A predator-prey system with stage structure for predator. Comput. Math. Appl., 33(8):83-91, 1997. http://dx.doi.org/10.1016/S0898-1221(97)00056-4.

[21] Y. Xiao, D. Cheng and H. Qin. Optimal impulsive control in periodic ecosystem. Systems Control Lett., 7(55):558-565, 2006. http://dx.doi.org/10.1016/j.sysconle.2005.12.003.

[22] T. Zhang, X. Meng and Y. Song. The dynamics of a high-dimensional delayed pest management model with impulsive pesticide input and harvesting prey at different fixed moments. Nonlinear Dynam., 64(1):1-12, 2011. http://dx.doi.org/10.1007/s11071-010-9840-1. 